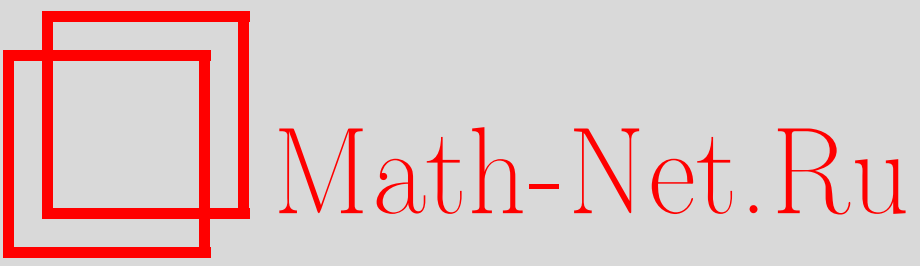

М. Д. Миссаров, Критические явления в фермионной иерархической модели, TMФ, 1998, том 117, номер 3, 471-488

DOI: https://doi.org/10.4213/tmf946

Использование Общероссийского математического портала Math-Net.Ru подразумевает, что вы прочитали и согласны с пользовательским соглашением

http://www.mathnet.ru/rus/agreement

Параметры загрузки:

IP: 54.197 .130 .99

26 апреля 2023 г., 18:21:01 


\author{
ТЕОРЕТИЧЕСКАЯ \\ И МАТЕМАТИЧЕСКАЯ \\ ФИЗИКА \\ Том 117, № 3 \\ декабрь, 1998
}

(C) 1998 г.

М. Д. Миссаров*

\title{
КРИТИЧЕСКИЕ ЯВЛЕНИЯ \\ В ФЕРМИОННОЙ ИЕРАРХИЧЕСКОЙ МОДЕЛИ
}

Обсуждаются некоторые результаты о динамике преобразования ренормализационной группы в пространстве констант связи фермионной иерархической модели. Критическое поведение в данной модели интерпретируется в терминах сложного поведения грассмановозначной плотности распределения среднего спина с подходящей нормировкой. Определяются и вычисляются некоторые критические индексы.

\section{1. ВВЕДЕНИЕ}

В работах [1-3] мы начали исследование иерархической фермионной модели методами ренормализационной групшы (РГ). В частности, было показано, что преобразование РГ Каданова-Вильсона сводится к некоторому рациональному преобразованию в плоскости констант связи, описаны неподвижные точки РГ-преобразования, устойчивые инвариантные кривые, проходяшие через эти точки, а также исследована глобальная динамика РГ в некоторых областях плоскости констант связи.

В данной работе мы дополняем результаты о динамике РГ-преобразований и даем описание критических явлений в этой модели.

Напомним основные определения. Иерархическая решетка задана как множество натуральных чисел $N$ с иерархическим расстоянием $d(i, j), \quad i, j \in N$, где $d(i, j)=n^{s(i, j)}$, если $i \neq j ; s(i, j)=\min \left\{s:\right.$ сушествует $k$ такое, что $\left.i \in V_{k, s}, j \in V_{k, s}\right\}$; $V_{k, s}=\left\{j: j \in N,(k-1) n^{s}<j \leqslant k n^{s}\right\} ; n$ - некоторое фиксированное натуральное число. В узлах этой решетки находятся четырехкомпонентные спины $\psi^{*}(i)=$ $\left(\bar{\psi}_{1}(i), \psi_{1}(i), \bar{\psi}_{2}(i), \psi_{2}(i)\right)$, компоненты которых являются образуюшими алгебры Грассмана.

Напомним (см. [1]), что мы рассматриваем фермионное поле в объеме $\Lambda_{N} \equiv$ $V_{1, N}$ с гиббсовским состоянием $\rho_{N}(r, g)$ (зависящим также от вешественного параметра $\alpha$ ) на грассмановой подалгебре $A_{N}$, порожденной $4 \cdot n^{N}$ образуюшими $\left(\psi_{1}(i), \bar{\psi}_{1}(i), \psi_{2}(i), \bar{\psi}_{2}(i)\right), \quad i \in \Lambda_{N}$.

Если $F\left(\psi^{*}\right) \in A_{N}$, то

$$
\rho_{N}(r, g)\left(F\left(\psi^{*}\right)\right)=Z_{N}^{-1} \int F\left(\psi^{*}\right) \exp \left\{-H_{N}\left(\psi^{*} ; r, g\right)\right\} d \psi^{*},
$$

\footnotetext{
* Казанский государственный университет, Казань, Россия
} 
где

$$
\begin{aligned}
d \psi^{*} & =\prod_{i \in \Lambda_{N}} d \psi_{1}(i) d \bar{\psi}_{1}(i) d \psi_{2}(i) d \bar{\psi}_{2}(i), \\
H_{N}\left(\psi^{*} ; r, g\right) & =H_{0, N}\left(\psi^{*}, \alpha\right)+\sum_{i \in \Lambda_{N}} L\left(\psi^{*}(i) ; r, g\right), \\
L\left(\psi^{*}(i) ; r, g\right) & =r\left(\bar{\psi}_{1}(i) \psi_{1}(i)+\bar{\psi}_{2}(i) \psi_{2}(i)\right)+g \bar{\psi}_{1}(i) \psi_{1}(i) \bar{\psi}_{2}(i) \psi_{2}(i), \\
H_{0, N}\left(\psi^{*} ; \alpha\right) & =\sum_{i, j \in \Lambda_{N}} d_{0, N}(i, j)\left(\bar{\psi}_{1}(i) \psi_{1}(j)+\bar{\psi}_{2}(i) \psi_{2}(j)\right), \\
d_{0, N}(i, j) & =\frac{1-n^{\alpha-1}}{1-n^{-\alpha}} d^{-\alpha}(i, j)-\frac{\left(1-n^{\alpha-1}\right)^{2} n^{-\alpha(N+1)}}{\left(1-n^{-\alpha}\right)\left(1-n^{-1}\right)}, \quad i \neq j, \\
d_{0, N}(i, i) & =\frac{1-n^{\alpha-2}}{1-n^{-1}}-\frac{\left(1-n^{\alpha-1}\right)^{2}}{\left(1-n^{-\alpha}\right)\left(1-n^{-1}\right)} n^{-\alpha(N+1)}, \\
Z_{N}(r, g) & =\int \exp \left\{-H_{N}\left(\psi^{*} ; r, g\right)\right\} d \psi^{*} .
\end{aligned}
$$

Интегрирование ведется по обычным правилам суперанализа [4]. РГ-преобразование определяется по формуле

$$
r(\alpha) \psi^{*}(i)=n^{-\alpha / 2} \sum_{j \in V_{i, 1}} \psi^{*}(j) .
$$

В [2] было показано, что

$$
\rho_{N}(r, g)\left(F\left(r(\alpha) \psi^{*}\right)\right)=\rho_{N-1}\left(r^{\prime}, g^{\prime}\right)\left(F\left(\psi^{*}\right)\right) .
$$

В пространстве констант связи $(r, g)$ РГ-преобразование действует как рациональное отображение

$$
\begin{aligned}
& r^{\prime}=n^{\alpha-1}\left(\frac{(r+1)^{2}-g}{(r+1)^{2}-\frac{g}{n}}(r+1)-1\right), \\
& g^{\prime}=n^{2 \alpha-3}\left(\frac{(r+1)^{2}-g}{(r+1)^{2}-\frac{g}{n}}\right)^{2} g,
\end{aligned}
$$

которое имеет нетривиальные ветви неподвижных точек $\left(r_{ \pm}, g_{ \pm}\right)$, где

$$
\begin{aligned}
r_{ \pm} & =\frac{ \pm \sqrt{n}-n^{\alpha-1}}{1 \mp \sqrt{n}}, \quad g_{ \pm}=g_{ \pm}\left(r_{ \pm}\right), \\
g_{ \pm}(r) & =\frac{r(1+r)^{2}}{1+r \pm \frac{1}{\sqrt{n}}} .
\end{aligned}
$$

В работе [3] было показано, что при $\alpha \geqslant 3 / 2$ компонента связности устойчивой РГ-инвариантной кривой, проходяшей через плюсовую (минусовую) неподвижную точку (НT) задается графиком гладкой, монотонно возрастаюшей (убываюшей) функции $g=$ $h_{+}(r), r>0\left(g=h_{-}(r), r<-1\right)$. При этом $h_{+}(r) \rightarrow 0$ при $r \rightarrow 0$ и $h_{-}(r) \rightarrow 0$ при 
$r \rightarrow-1, h_{+}(r) \rightarrow+\infty$ при $r \rightarrow+\infty$ и $h_{-}(r) \rightarrow+\infty$ при $r \rightarrow-\infty$. При $1<\alpha \leqslant 3 / 2$ кривая $g=h_{+}(r)$ уже является устойчивой РГ-инвариантной кривой для нулевой НТ $(r=0, g=0)$.

Кривая $g=h_{+}(r)$ лежит в области

$$
\begin{aligned}
G_{1}= & \left\{(r, g): 0 \leqslant r \leqslant r_{+}, \max \left\{0, g_{4}(r), g_{1}^{+}(r)\right\} \leqslant g \leqslant g_{+}(r)\right\} \cup \\
& \cup\left\{(r, g): \max \left\{0, r_{+}\right\} \leqslant r, g_{+}(r) \leqslant g \leqslant g_{1}^{+}(r)\right\},
\end{aligned}
$$

кривая $g=h_{-}(r)-$ в области

$$
\begin{aligned}
G_{2}= & \left\{(r, g): r \leqslant r_{-}, g_{1}^{-}(r) \leqslant g \leqslant g_{-}(r)\right\} \cup \\
& \cup\left\{(r, g): r_{-}<r<-1, g_{-}(r)<g<g_{1}^{-}(r)\right\},
\end{aligned}
$$

здесь

$$
\begin{aligned}
g_{1}^{ \pm}(r) & =\frac{(r+1)(n \mp \sqrt{n})-n+n^{2-\alpha}}{(r+1)(n \mp \sqrt{n})-1+n^{1-\alpha}}(r+1)^{2}, \\
g_{4}(r) & =\frac{r\left(1-n^{\alpha-2}\right)}{r\left(1-n^{\alpha-2}\right)+1-n^{-1}}(r+1)^{2} .
\end{aligned}
$$

Обозначим кривые $g=h_{+}(r), 0 \leqslant r<\infty$, и $g=h_{-}(r),-\infty<r<-1$, через $\gamma_{1}$ и $\gamma_{2}$, соответственно. В работе [2] было показано, что области верхней полуплоскости, лежашие справа от $\gamma_{1}$ и слева от $\gamma_{2}$, являются РГ-инвариантными и найдена асимптотика итераций РГ-преобразования в этих областях. Обозначим эти области через $\Omega_{1}$ и $\Omega_{2}$, соответственно.

В разделе 2 мы исследуем вопрос о динамике РГ в области между кривыми $\gamma_{1}$ и $\gamma_{2}$ при $2>\alpha>1$. Будет доказано, что при $r \geqslant-\left(n^{\alpha}-n^{\alpha-1}\right)\left(n^{\alpha}-1\right)^{-1}$ и достаточно больших $g$ точка $(r, g)$ за конечное число РГ-итераций попадает в область $\Omega_{2}$, а при $r<-\left(n^{\alpha}-n^{\alpha-1}\right)\left(n^{\alpha}-1\right)^{-1}$ и достаточно больших $g-$ в область $\Omega_{1}$. Компьютерные эксперименты показывают, что в области между $\gamma_{1}$ и $\gamma_{2}$ располагаются все остальные части устойчивых инвариантных кривых для плюсовой и минусовой НТ, а также устойчивая инвариантная кривая для бесконечно удаленной НТ. Все эти кривые расслаивают область между $\gamma_{1}$ и $\gamma_{2}$ на бесконечное число связных областей, точки из которых при РГ-итерациях попадают либо в область $\Omega_{1}$, либо в область $\Omega_{2}$, причем все эти области чередуются между собой фрактальным образом.

В разделе 3 результаты о глобальной динамике РГ используются для описания критических явлений в данной модели. Нетривиальность критических явлений будет следовать из анализа предельного поведения грассмановозначной плотности распределения суммарного спина с подходящей нормировкой

$$
q_{N}^{(a)}\left(x^{*} ; r, g\right)=\rho(r, g) \delta\left(\frac{1}{n^{a N}} \sum_{i \in \Lambda_{N}} \psi^{*}(i)-x^{*}\right),
$$

где $\rho(r, g)$ - термодинамический предел состояний $\rho_{M}(r, g)$ при $M \rightarrow \infty, \delta$ - грассманова дельта-функция, $x^{*}=\left(x_{1}, \bar{x}_{1}, x_{2}, \bar{x}_{2}\right)$, где $x_{1}, \bar{x}_{1}, x_{2}, \bar{x}_{2}$ - грассмановы переменные. 
Показано, что если $R^{N_{0}}(\alpha)(r, g)$ попадает в $\Omega_{1}$ или $\Omega_{2}$ при некотором $N_{0} \geqslant 0$, где $R(\alpha)(r, g)=\left(r^{\prime}, g^{\prime}\right)$ задается формулами (6), то при нормировке $a=1 / 2$ имеем

$$
\lim _{N \rightarrow \infty} q_{N}^{1 / 2}\left(x^{*} ; r, g\right)=\frac{1}{c_{1}^{2}(r, g)} \exp \left\{-c_{1}(r, g)\left(\bar{x}_{1} x_{1}+\bar{x}_{2} x_{2}\right)\right\} .
$$

Здесь $c_{1}(r, g)$ определяется как предел

$$
c_{1}^{2}(r, g)=\lim _{N \rightarrow \infty} r^{(N)} n^{-N(\alpha-1)},
$$

где $r^{(N)}$ определяется соотношением $\left(r^{(N)}, g^{(N)}\right)=R^{N}(\alpha)(r, g)$. Существование константы $c_{1}(r, g)$ было доказано в работе [2].

Другими словами, предел задается "гауссовским" распределением, причем $c_{1}(r, g)>0$ в случае, если $(r, g)$ лежит в зоне притяжения $\Omega_{1}$, и $c_{1}(r, g)<0$, если $(r, g)$ лежит в зоне притяжения $\Omega_{2}$.

Если мы рассмотрим луч $(r, g), g>0$, то, например, при фиксированном $r>0$ и при возрастаюшем $g$ мы наблюдаем перестройку предельной плотности. При достаточно малых и достаточно больших $g$ предельная плотность является гауссовской (но с разным знаком у константы связи). Сушествует также область значений $g$, в которой предельная плотность бесконечно много раз (но на множестве меры 0) становится негауссовской и на множестве полной меры является гауссовской, бесконечно много раз чередуя знак константы связи.

Если же $(r, g)$ лежит на инвариантной кривой $\gamma_{1}\left(\gamma_{2}\right)$, то нужна нестандартная нормировка $a=\alpha / 2$, и в этом случае предельное поведение $q_{N}^{\alpha / 2}\left(x^{*} ; r, g\right)$ является негауссовским и задается плотностью, соответствуюшей плюсовой (или минусовой) НТ.

Поведение $c_{1}(r, g)$ имеет особенность при $g \rightarrow g_{\text {кр }}(r)$, где $\left(r, g_{\text {кр }}(r)\right)$ - точка пересечения полупрямой $(r, g), g>0$, с инвариантной кривой $\gamma_{1}$ (или $\gamma_{2}$ ), что позволяет определить еще один критический индекс в этой модели.

\section{2. ДИНАМИКА РЕНОРМАЛИЗАЦИОННОЙ ГРУППЫ В ОБЛАСТИ МЕЖДУ КРИВЫМИ $\gamma_{1}$ И $\gamma_{2}$}

В дальнейшем нам будет удобно наряду с переменными $r$ и $g$ использовать обозначения

И

$$
\beta=\beta(r, g)=\frac{g}{(r+1)^{2}}
$$

$$
\sigma=\sigma(r, g)=\frac{1-\beta(r, g)}{1-\frac{\beta(r, g)}{n}} .
$$

Мы также будем использовать обозначения $\lambda_{1}=n^{\alpha-1}, \lambda_{2}=n^{2 \alpha-3}$. Заметим, что $\lambda_{1}, \lambda_{2}$ являются собственными числами дифференциала отображения $R(\alpha)$ в нуле.

В работе [3] было доказано следуюшее утверждение (лемма 3 ): парабола $g(r)=\beta(r+$ $1)^{2}, \beta=$ const, за одну итерацию РГ переходит в параболу $g^{\prime}\left(r^{\prime}\right)=\beta\left(r^{\prime}+\lambda_{1}\right)^{2} / n$, где $r^{\prime}$ линейно зависит от $r, r^{\prime}=k r+b$, где $k>0$ при $\beta<1$ и $\beta>n, k<0$ при $1<\beta<n$. Точки пересечения парабол $g(r)$ и $g^{\prime}(r)$ лежат на прямых $r=r_{ \pm}$. 
Отсюда следует, что любая точка $(r, g)$ такая, что $r>-1, n>\beta(r, g) \geqslant 1$, за одну итерацию РГ переходит в область $g<\left(r+\lambda_{1}\right)^{2}, r \leqslant-\lambda_{1}$. Из теоремы об инвариантных кривых видно, что она попадает в область $\Omega_{2}$.

Изучим теперь вопрос о том, куда переходят точки прямой $r=r_{0}$. Справедлива следуюшая лемма.

ЛЕмма 1. Прямая $r=r_{0}$ за одну итерацию РГ переходит в кривую

$$
g=\frac{r-\lambda_{1} r_{0}}{r-\lambda_{1} n r_{0}-\lambda_{1}(n-1)}\left(r+\lambda_{1}\right)^{2} .
$$

При $r_{0}>-1 \quad\left(r_{0}<-1\right)$ точки $\left(r_{0}, g\right)$ такие, что $\beta\left(r_{0}, g\right)>n$ отображаются на ту часть кривой (11), для которой $r>\lambda_{1} n r_{0}+\lambda_{1}(n-1) \quad\left(r<\lambda_{1} n r_{0}+\lambda_{1}(n-1)\right.$, соответственно). Кроме того, при $g \rightarrow+\infty$

$$
r^{\prime}\left(r_{0}, g\right) \rightarrow \lambda_{1} n r_{0}+\lambda_{1}(n-1), \quad g^{\prime}\left(r_{0}, g\right) \rightarrow \infty
$$

В дополнение к этой лемме заметим, что прямая $r=-1$ переходит в прямую $r=-\lambda_{1}$ с сохранением всех остальных утверждений леммы 1.

Из этой леммы видно, что при

$$
r \geqslant-\frac{\lambda_{1}(n-1)}{\lambda_{1} n-1}
$$

точки $(r, g), \beta(r, g)>n$ уходят направо по $r: r^{\prime}(r, g)>r$.

Также имеет место лемма 2.

ЛЕмма 2. Если $r$ удовлетворяет условию (12) и $\beta(r, g)>n$, то за конечное число итераций РГ точка $(r, g)$ попадает в область $\Omega_{2}$.

ДокАЗАТЕЛЬСтво. Воспользуемся соотношением

$$
\beta^{\prime}=\beta\left(r^{\prime}, g^{\prime}\right)=\frac{\beta}{n}\left(1-\frac{\lambda_{1}-1}{\lambda_{1} \sigma(r+1)}\right)^{-2} .
$$

При $\beta>n$ имеем $\sigma=(1-\beta)(1-\beta / n)^{-1}>n$, а из (12) следует

$$
1>\frac{\lambda_{1}-1}{\lambda_{1} \sigma(r+1)},
$$

так что $\beta^{\prime}>1$. Кроме того, из (13) вытекает, что при достаточно больших $r$

$$
\beta^{\prime}<\frac{3 \beta}{2 n}
$$

Так как при $\beta>n$ имеем

$$
r^{\prime}=\lambda_{1}\left(\frac{1-\beta}{1-\frac{\beta}{n}}(r+1)-1\right)>\lambda_{1}(n(r+1)-1),
$$


то при некотором $k$ либо точка $\left(r^{(k)}, g^{(k)}\right)$ попадает в область

$$
(r+1)^{2}<g<n(r+1)^{2}, \quad r>-1,
$$

а оттуда в область $\Omega_{2}$, либо она попадает в зону действия неравенства (14) и, следовательно, через некоторое дополнительное число итераций попадает в область (15).

Рассмотрим теперь случай, когда $r<-\lambda_{1}(n-1)\left(\lambda_{1} n-1\right)^{-1}$. В дальнейшем нам понадобится семейство кривых $g=f(r ; a)$, где

$$
f(r ; a)=\frac{r}{r-a}(r+1)^{2} .
$$

В частности, при $a=-\left(1+n^{-1 / 2}\right)$ получаем $f(r ; a)=g_{+}(r)$.

Непосредственным вычислением проверяется, что РГ-образ кривой $g=f(r ; a)$ есть кривая $g=f^{\prime}(r ; a)$, где

$$
f^{\prime}(r ; a)=\frac{r\left(r+\lambda_{1}\right)^{2}}{r-\lambda_{1} n a-\lambda_{1}(n-1)} .
$$

Докажем следуюшую лемму.

Лемма 3. Пусть $r<-\left(1+n^{-1 / 2}\right), \quad g \geqslant g_{+}(r)$. За конечное число итераций точка $(r, g)$ попадает в область $\Omega_{1}$.

ДоКАЗАТЕЛЬСТво. Заметим, во-первых, что любая точка, удовлетворяющая условиям леммы, лежит на некоторой кривой $g=f(r ; a), a<-\left(1+n^{-1 / 2}\right)$. Обозначим через $r_{1}(a)$ абсциссу точки пересечения кривой $g=f(r ; a)$ с параболой $g=n(r+1)^{2}$ :

$$
r_{1}(a)=\frac{a n}{n-1} .
$$

Из соотношения

$$
r^{\prime}=-\lambda_{1}\left(1+r_{1}(a)\right) \frac{r}{r-r_{1}(a)}
$$

видно, что участок кривой $g=f(r ; a), r<r_{1}(a)$, при РГ-преобразовании отобразится на участок кривой $g=f^{\prime}(r ; a), r>r_{2}(a)$, где $r_{2}(a)=-\lambda_{1}\left(1+r_{1}(a)\right)$.

Заметим, что при $r>0$ выполнено $f^{\prime}\left(r ; a_{1}\right)>f^{\prime}\left(r ; a_{2}\right)>0$, если $a_{2}<a_{1}<1 / n-1$, и что $r_{2}\left(a_{2}\right)>r_{2}\left(a_{1}\right)$ при $a_{1}>a_{2}$. Поэтому, если мы докажем, что участок кривой

$$
g=f^{\prime}\left(r ;-\left(1+n^{-1 / 2}\right)\right), \quad r>r_{2}\left(-\left(1+n^{-1 / 2}\right)\right)
$$

лежит справа от кривой $\gamma_{1}$, то то же самое будет верно и для участка кривой $g=f^{\prime}(r ; a)$, $r>r_{2}(a)$, если $a<-\left(1+n^{-1 / 2}\right)$.

Поскольку $f\left(r ;-\left(1+n^{-1 / 2}\right)\right)=g_{+}(r)$, то

$$
f^{\prime}\left(r ;-\left(1+n^{-1 / 2}\right)\right)=g_{+}^{\prime}(r)=\frac{r}{r+\lambda_{1}\left(1+n^{1 / 2}\right)}\left(r+\lambda_{1}\right)^{2} .
$$


Мы имеем следуюшее разложение:

$$
\begin{aligned}
g_{+}(r)-g_{+}^{\prime}(r)= & \frac{\left(n^{1 / 2}-1\right)\left(\lambda_{1}+n^{-1 / 2}\right)}{\left(r+1+n^{-1 / 2}\right)\left(r+\lambda_{1}\left(1+n^{1 / 2}\right)\right)} \times \\
& \times\left(r+\frac{n^{1 / 2}-\lambda_{1}}{n^{1 / 2}-1}\right)\left(r+\left(1+n^{-1 / 2}\right) \frac{\lambda_{1}}{\lambda_{1}+n^{-1 / 2}}\right) .
\end{aligned}
$$

Поскольку

$$
r_{2}\left(-\left(1+n^{-1 / 2}\right)\right)=\lambda_{1}\left(n^{-1 / 2}-1\right)^{-1}>r_{+}(\alpha)=\left(\lambda_{1}-n^{1 / 2}\right)\left(n^{1 / 2}-1\right)^{-1},
$$

то из $(20)$ видно, что при $r>r_{2}\left(-\left(1+n^{-1 / 2}\right)\right)$ имеет место $g_{+}(r)>g_{+}^{\prime}(r)$, а это означает, что $g_{+}^{\prime}(r)$ лежит справа от $\gamma_{1}$.

Таким образом, мы доказали, что любая точка из области

$$
A_{1}=\left\{(r, g): r<r_{1}\left(-\left(1+n^{-1 / 2}\right)\right), f\left(r ;-\left(1+n^{-1 / 2}\right)\right)<g<n(r+1)^{2}\right\}
$$

за одну РГ-итерацию попадет в область $\Omega_{1}$.

Рассмотрим теперь точки, лежащие в области

$$
A_{2}=\left\{(r, g): r<-\left(1+n^{-1 / 2}\right), g>\max \left(n(r+1)^{2}, f\left(r ;-\left(1+n^{-1 / 2}\right)\right)\right)\right\} .
$$

Легко проверить, что РГ-итерация отображает участок кривой $g=f(r ; a)$, $r_{1}(a)<r<a$ на участок кривой $g=f^{\prime}(r ; a), \quad r<\lambda_{1}(n a+n-1)$, причем $f^{\prime}(r ; a)>$ $f(r ; a)$ при $r<\lambda_{1}(n a+n-1)$. Из этого факта и формулы (13) следует, что за конечное число РГ-итераций любая точка из области $A_{2}$ попадет в область $A_{1}$.

Необходимо сделать следуюшее замечание. Как видно из формул, задаюших преобразование РГ, оно не определено для точек параболы $g=n(r+1)^{2}$. Это ограничение можно преодолеть, если определить действие РГ в пространстве коэффициентов разложения грассмановозначной плотности распределения единичного спина. Произвольная ненормированная плотность спина в нашей модели задается в виде

$$
P\left(\bar{\psi}_{1}, \psi_{1}, \bar{\psi}_{2}, \psi_{2}\right)=c_{0}+c_{1}\left(\bar{\psi}_{1} \psi_{1}+\bar{\psi}_{2} \psi_{2}\right)+c_{2}\left(\bar{\psi}_{1} \psi_{1} \bar{\psi}_{2} \psi_{2}\right),
$$

где $\bar{\psi}_{1}, \psi_{1}, \bar{\psi}_{2}, \psi_{2}$ - образуюшие алгебры Грассмана, задаюшие спин в фиксированном узле решетки, и $\left(c_{0}, c_{1}, c_{2}\right)$ предполагается 3 -мерным вешественным вектором. В частности, плотности

$$
\exp \left\{-r\left(\bar{\psi}_{1} \psi_{1}+\bar{\psi}_{2} \psi_{2}\right)-g \bar{\psi}_{1} \psi_{1} \bar{\psi}_{2} \psi_{2}\right\}
$$

соответствует набор коэффициентов $\left(1,-r, r^{2}-g\right)$, а $\delta$-функции $\delta(\psi)=\bar{\psi}_{1} \psi_{1} \bar{\psi}_{2} \psi_{2}$ - набор $(0,0,1)$. Если мы будем описывать плотность как точку 2-мерного проективного пространства $\left(c_{0}, c_{1}, c_{2}\right)$, то можно показать, что действие РГ в пространстве констант $\left(c_{0}, c_{1}, c_{2}\right)$ задается следующим образом:

$$
\begin{aligned}
& c_{0}^{\prime}=\left(c_{1}-c_{0}\right)^{2}-\frac{1}{n}\left(c_{1}^{2}-c_{0} c_{2}\right), \\
& c_{1}^{\prime}=\lambda_{1}\left(\left(c_{1}-c_{0}\right)\left(c_{2}-c_{1}\right)-\frac{1}{n}\left(c_{1}^{2}-c_{0} c_{2}\right)\right), \\
& c_{2}^{\prime}=\lambda_{1}^{2}\left(\left(c_{2}-c_{1}\right)^{2}-\frac{1}{n}\left(c_{1}^{2}-c_{0} c_{2}\right)\right) .
\end{aligned}
$$


Как преобразование $P^{2}$ в себя это отображение определено всюду, кроме точки $(1,1,1)$. Параболе $g=n(r+1)^{2}$ в новых координатах отвечает кривая $\left(1,-r, r^{2}-n(r+1)^{2}\right)$, $r \neq-1$, которая за одну итерацию преобразования (21) переходит в кривую

$$
\left(0,1, \lambda_{1}(n-1)\left(r+\frac{n+1}{n-1}\right)\right),
$$

которая не описывается в координатах $(r, g)$. Однако еше за одну итерацию преобразования (21) кривая (22) переходит в кривую

$$
\left(1, \lambda_{1} \frac{t(r)-1-n^{-1}}{1-n^{-1}}, \lambda_{1}^{2} \frac{(t(r)-1)^{2}-n^{-1}}{1-n^{-1}}\right)
$$

где $t(r)=\lambda_{1}(n-1)\left(r+(n+1)(n-1)^{-1}\right)$. Таким образом, возврашаясь к координатам $(r, g)$, мы видим, что две итерации РГ-преобразования переводят параболу $g=n(r+1)^{2}$ в параболу

$$
\begin{aligned}
& g^{(2)}=\frac{1}{n}\left(r^{(2)}+\lambda_{1}\right)^{2} \\
& r^{(2)}=-\lambda_{1}^{2} n r-\lambda_{1}\left(\lambda_{1} n-1\right) \frac{n+1}{n-1} .
\end{aligned}
$$

Легко видеть, что при таком определении РГ-преобразования часть параболы $g=$ $n(r+1)^{2}, r<r_{1}\left(-\left(1+n^{-1 / 2}\right)\right)$, за две итерации перейдет в область $\Omega_{1}$.

Таким образом, лемму 3 можно считать полностью доказанной.

Что касается области $-\left(1+n^{-1 / 2}\right)<r<-\lambda_{1}(n-1)\left(\lambda_{1} n-1\right)^{-1}$, то мы ограничимся следующей леммой.

ЛЕмма 4. Если $-\left(1+n^{-1 / 2}\right) \leqslant r<-\lambda_{1}(n-1)\left(\lambda_{1} n-1\right)^{-1}$ и g достаточно велико, то за конечное число РГ-итераций точка $(r, g)$ попадет в область $\Omega_{1}$.

ДокАЗАТЕЛЬСтво. Пусть $s(t)=(r(t), g(t))$ - непрерывная кривая, параметризованная параметром $t, 0<t<b$, асимптотически стремяшаяся к некоторой вертикальной прямой $r=r_{0}: r(t) \rightarrow r_{0}, g(t) \rightarrow \infty$ при $t \rightarrow 0$. Предположим также, что кривая $s(t)$ лежит в области $g>n(r+1)^{2}$. Тогда РГ-образ этой кривой $f^{\prime}(t)=\left(r^{\prime}(t), g^{\prime}(t)\right)$ также является непрерывной кривой от $t$, асимптотически стремящейся к прямой $r=$ $r_{1}(0): r^{\prime}(t) \rightarrow r_{1}, g^{\prime}(t) \rightarrow \infty$ при $t \rightarrow 0$. Здесь $r_{1}=\lambda_{1}\left(n\left(r_{0}+1\right)-1\right)$. Можно выбрать $b_{1}<b$ так, чтобы при $0<t<b_{1}$ кривая $f^{\prime}(t)$ лежала в области $g>n(r+1)^{2}$. Пусть $r_{k}=\lambda_{1}\left(n\left(r_{k-1}+1\right)-1\right)$. Если $r_{0}<-\lambda_{1}(n-1)\left(\lambda_{1} n-1\right)^{-1}$, то легко видеть, что за конечное число шагов $k$ величина $r_{k}$ окажется меньше $-\left(1+n^{-1 / 2}\right)$. Взяв в качестве начальной кривой вертикальную прямую $r=r_{0}$ и применяя вышеприведенное рассуждение несколько раз, мы получим утверждение леммы.

Мы использовали компьютер для исследования динамики РГ в тех областях верхней полуплоскости, на которые не распространяются утверждения вьшеприведенных лемм. Приведем некоторые результаты. 


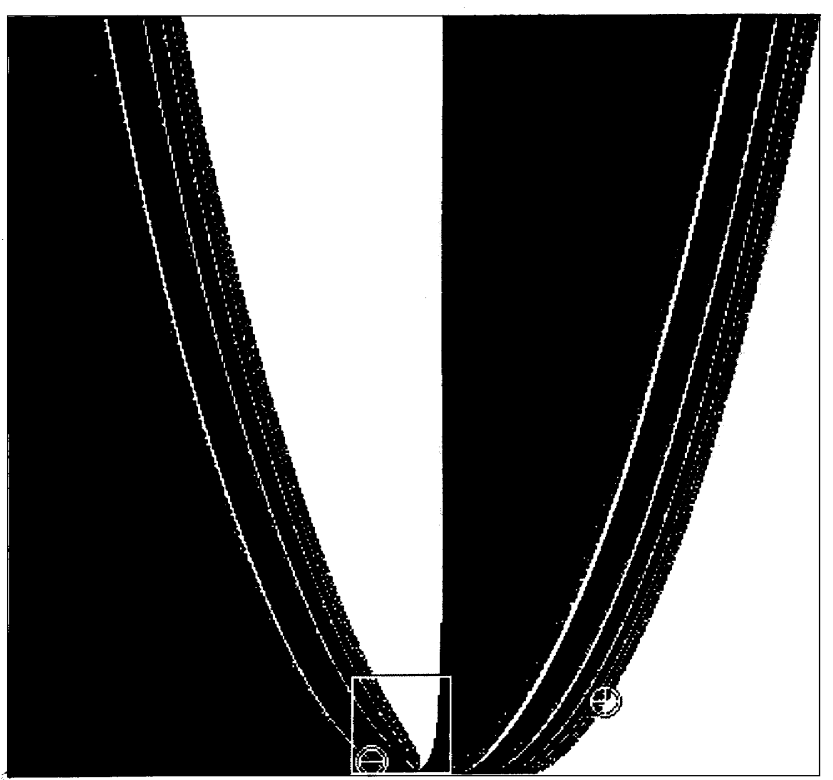

Рис. 1

Из точки $(-1,0)$ выходит кривая, асимптотически стремящаяся к прямой $r_{0}=$ $-\lambda_{1}(n-1)\left(\lambda_{1} n-1\right)^{-1}$, которую, по-видимому, также можно считать графикиом гладкой монотонно возрастаюшей функции $g=h(r), r_{0}<-\lambda_{1}(n-1)\left(\lambda_{1} n-1\right)^{-1}$. Эта кривая является частью устойчивой инвариантной кривой для бесконечно удаленной НТ (в проективных координатах она задается набором $(0,0,1))$. Обозначим эту кривую $\gamma_{\infty}$. Пусть

$$
\tilde{\gamma}_{i}=\bigcup_{n=0}^{\infty} R^{-n} \gamma_{i}, \quad i=1,2, \infty
$$

т.е. $\tilde{\gamma}_{1}$ - вся устойчивая инвариантная кривая для плюсовой НТ, $\tilde{\gamma}_{2}-$ для минусовой НТ, $\tilde{\gamma}_{\infty}$ - для бесконечно удаленной НТ.

В проективных координатах каждая кривая $\gamma_{i}$ является гладкой связной кривой, бесконечно много раз проходяшей через точки $(-1,0)$ и ее РГ-прообразы $\left(-\lambda_{1}^{-k}, 0\right), \quad k=$ $1,2, \ldots$.

В координатах $(r, g)$ эти кривые несвязны и являются объединением своих связных частей, каждая из которых проходит только через одну из точек вида $\left(-\lambda_{1}^{-k}, 0\right), k=$ $0,1,2, \ldots$ Отметим, что вся группа РГ-преобразований не определена в точке $(-1,0)$ и ее прообразах и поэтому их надо, вообще говоря, выколоть из кривых $\tilde{\gamma}_{i}, i=1,2, \infty$.

Связность кривых $\tilde{\gamma}_{i}$ можно увидеть в проективных координатах: соединение связных частей между собой происходит на множестве точек вида $\left(0, c_{1}, c_{2}\right)$, которое не изображается в координатах $(r, g)$. По-видимому, почти все точки верхней полуплоскости в области между кривыми $\gamma_{1}$ и $\gamma_{2}$, не лежашие на кривых $\tilde{\gamma}_{1}, \tilde{\gamma}_{2}, \tilde{\gamma}_{\infty}$, за конечное число итераций РГ попадают в области $\Omega_{1}$ или $\Omega_{2}$. Рассмотрим вертикальную прямую 


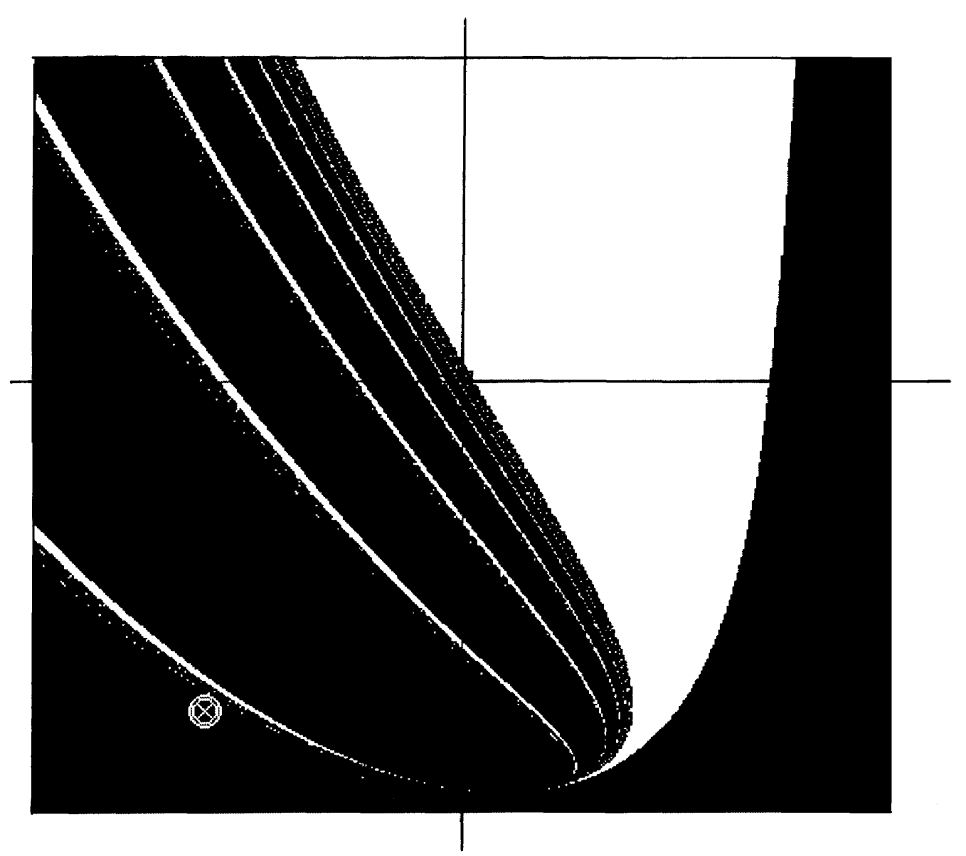

Рис. 2

$r=r_{0}, g>0$. Если $-\lambda_{1}(n-1)\left(\lambda_{1} n-1\right)^{-1}<r_{0}<c_{1}, c_{1}<-\lambda_{1}^{-1}$, то вертикальная прямая пересечет кривую $\gamma_{\infty}$ в некоторой точке $\left(r_{0}, g_{0}\right)$ и разобьется на два интервала:

$$
I_{1}=\left\{r=r_{0}, 0<g<g_{0}\right\} \quad \text { и } \quad I_{2}=\left\{r=r_{0}, g_{0}<g<\infty\right\} .
$$

Все точки из $I_{1}$ за конечное число итераций РГ попадают в область $\Omega_{2}$, а все точки из $I_{2}-$ в область $\Omega_{1}$. Для всех остальных значений $r_{0}$ луч $r=r_{0}, g>0$ бесконечно много раз пересекает кривые $\tilde{\gamma}_{1}, \tilde{\gamma}_{2}, \tilde{\gamma}_{\infty}$. Если $r_{0}>0$, то луч $\left(r=r_{0}, g>0\right)$ разбивается на интервалы $I_{1}=\left\{r=r_{0}, 0<g<g_{0}\left(r_{0}\right)\right\}, I_{2}\left\{r=r_{0}, g_{1}\left(r_{0}\right)<g<\infty\right\}$ и бесконечное множество интервалов, фрактальным образом разбиваюших отрезок $\left(r=r_{0}, g_{0}\left(r_{0}\right)<\right.$ $\left.g<g_{1}\left(r_{0}\right)\right)$. Точки из $I_{1}$ лежат в $\Omega_{1}$, точки из $I_{2}$ за конечное число РГ-итераций попадают в $\Omega_{2}$, а поведение РГ в остальных интервалах чередуется. При $r_{0}<-1$ картина аналогичная с той лишь разницей, что точки из $I_{1}$ лежат в $\Omega_{2}$, а точки из $I_{2}$ за конечное число РГ-итераций попадают в $\Omega_{1}$. Такая же картина имеет место и при $c_{1}<r_{0}<0$, но при этом как точки из $I_{1}$, так и точки из $I_{2}$ попадают в область $\Omega_{2}$ за конечное число РГ-итераций.

Для иллюстрации вышесказанного рассмотрим рис. 1 и 2. На рис. 1 изображена верхняя полуплоскость $g>0$. Часть полуплоскости окрашена черным цветом, часть - белым в соответствии со следующим правилом: черные точки при итерациях РГ уходят налево в область $\Omega_{2}$, белые точки при итерациях РГ уходят направо в область $\Omega_{1}$. Кружками на рис. 1 обведены плюсовая и минусовая НТ. Можно предположить, что почти все точки, лежашие на границе черной и белой областей, лежат на инвариантных кривых $\tilde{\gamma}_{1}$, $\tilde{\gamma}_{2}, \tilde{\gamma}_{\infty}$. Достаточно малая окрестность точки, лежашей на инвариантной кривой, де- 
лится этой кривой на две части. При этом различные инвариантные кривые характеризуются следуюшим образом. Для кривой $\tilde{\gamma}_{1}$ одна часть окрестности белая (состоит из белых точек), другая смешанная (состоит из белых и черных точек). Для кривой $\tilde{\gamma}_{\infty}$ одна часть окрестности белая, другая черная. Для кривой $\tilde{\gamma}_{2}$ одна часть окрестности черная, другая смешанная.

Участки кривых $\tilde{\gamma}_{1}$ и $\tilde{\gamma}_{\infty}$ видны на рисунке. Примеры участков кривой $\tilde{\gamma}_{2}$ не видны, но их сушествование проясняется при многократном увеличении изображения. На рис. 2 дано увеличенное изображение квадрата, выделенного на рис. 1. Центр кружка на рис. 2 изображает минусовую НТ, которая как раз лежит на кривой $\tilde{\gamma}_{2}$. В изображенном примере параметр РГ $\alpha=1.7, n=2$.

\section{3. КРИТИЧЕСКИЕ ЯВЛЕНИЯ}

Картину критических явлений в нашей модели проще всего описать, исследуя предельное поведение грассмановозначной "плотности" распределения нормированного подходяшим образом суммарного спина в объеме $\Lambda_{N}$, усредненного по состоянию $\rho_{N}$.

Обозначим через $\tilde{q}_{N}^{(a)}\left(x^{*} ; r, g\right)$ "плотность" распределения нормированного суммарного спина

$$
\psi_{N, a}^{*}=\frac{1}{n^{a N}} \sum_{i \in \Lambda_{N}} \psi^{*}(i)
$$

для поля $\psi^{*}$, заданного состоянием $\rho_{N}(\rho, g)$ :

$$
\tilde{q}_{N}^{(a)}\left(x^{*} ; r, g\right)=\rho_{N}(r, g)\left(\delta\left(\psi_{N, a}^{*}-x^{*}\right)\right),
$$

$x^{*}=\left(x_{1}, \bar{x}_{1}, x_{2}, \bar{x}_{2}\right)$ (напомним, что $\delta$-функция в анализе с антикоммутируюшими переменными имеет представление $\left.\delta\left(\psi^{*}\right)=\psi_{1} \bar{\psi}_{1} \psi_{2} \bar{\psi}_{2}\right)$.

Из результатов, приведенных в работы [1], получаем, что

$$
\begin{aligned}
\tilde{q}_{N}^{a}\left(x^{*} ; r, g\right) & =\rho_{N}(r, g)\left(\delta\left(n^{\left(\frac{\alpha}{2}-a\right) N} n^{-\frac{\alpha}{2} N} \sum_{i \in \Lambda_{N}} \psi^{*}(i)-x^{*}\right)\right)= \\
& =n^{4\left(\frac{\alpha}{2}-a\right) N} \tilde{q}_{0}^{(a)}\left(n^{\left(a-\frac{\alpha}{2}\right) N} x^{*} ; r^{(N)}, g^{(N)}\right)
\end{aligned}
$$

где $\left(r^{(N)}, g^{(N)}\right)=R^{N}(\alpha)(r, g)-N$-я итерация преобразования РГ $R(\alpha): R(\alpha)(r, g)=$ $\left(r^{\prime}, g^{\prime}\right)$, заданного соотношением (6).

Таким образом, мы можем вычислить $\tilde{q}_{N}^{(a)}\left(x^{*} ; r, g\right)$ явно:

$$
\tilde{q}_{N}^{(a)}\left(x^{*} ; r, g\right)=\frac{n^{(2 \alpha-4 a) N}}{\left(r^{N}+c_{0}\right)^{2}-g^{N}} \exp \left\{-L\left(x^{*} ; n^{(2 a-\alpha) N} r^{(N)}, n^{(4 a-2 \alpha) N} g^{(N)}\right)\right\},
$$

где (см. формулу (5))

$$
c_{0}=c_{0}(\alpha) \equiv d_{0,0}(i, i)=\left(1-n^{\alpha-2}\right)\left(1-n^{-1}\right)^{-1} .
$$

6 Теоретическая и математическая физика, т. 117, № 3, 1998 г. 
Прежде чем сформулировать теорему, напомним, что из результатов теоремы 2 работы [2] следует сушествование констант $c_{1}(r, g), c_{2}(r, g),(r, g) \in \Omega_{1} \cup \Omega_{2}$ таких, что

$$
\lim _{N \rightarrow \infty} r^{(N)} n^{-N(\alpha-1)}=c_{1}(r, g), \quad \lim _{N \rightarrow \infty} g^{(N)} n^{-N(2 \alpha-3)}=c_{2}(r, g) .
$$

При этом $c_{1}(r, g)>0$ для $(r, g) \in \Omega_{1}, c_{1}(r, g)<0$ для $(r, g) \in \Omega_{2}, c_{2}(r, g)=0$ только при $g=0$. Эти результаты справедливы для всех $\alpha>1$. Аналогичные результаты справедливы и для нижней полуплоскости.

Если плотность распределения спина может быть записана в экспоненциальной форме как

$$
p\left(x^{*} ; r, g\right)=\frac{1}{r^{2}-g} \exp \left\{-L\left(x^{*} ; r, g\right)\right\}
$$

то мы будем говорить, что

$$
\lim _{m \rightarrow \infty} p\left(x^{*} ; r_{m}, g_{m}\right)=p\left(x^{*} ; r, g\right),
$$

если $\lim _{m \rightarrow \infty}\left(r_{m}, g_{m}\right)=(r, g)$.

ТЕОРема 1. Пусть $2>\alpha>1$. Ecли $\left(r^{\left(N_{0}\right)}, g^{\left(N_{0}\right)}\right) \in \Omega_{1} \cup \Omega_{2}$ для некоторого $N_{0} \geqslant 0, m o$

$$
\lim _{N \rightarrow \infty} \tilde{q}_{N}^{(1 / 2)}\left(x^{*} ; r, g\right)=p\left(x^{*} ; c_{1}(r, g), 0\right) .
$$

$\operatorname{Ec} \Omega u(r, g) \in \tilde{\gamma}_{2}, m o$

$$
\lim _{N \rightarrow \infty} \tilde{q}_{N}^{(\alpha / 2)}\left(x^{*} ; r, g\right)=p\left(x^{*} ; c_{0}(\alpha)+r_{-}(\alpha), g_{-}(\alpha)\right) .
$$

$\operatorname{Ec} \Omega u(r, g) \in \tilde{\gamma}_{1}$, mo при $\alpha>3 / 2$

$$
\lim _{N \rightarrow \infty} \tilde{q}_{N}^{(\alpha / 2)}\left(x^{*} ; r, g\right)=p\left(x^{*} ; c_{0}(\alpha)+r_{+}(\alpha), g_{+}(\alpha)\right),
$$

a при $3 / 2 \geqslant \alpha>1$

$$
\lim _{N \rightarrow \infty} \tilde{q}_{N}^{(\alpha / 2)}\left(x^{*} ; r, g\right)=p\left(x^{*} ; c_{0}(\alpha)\right) .
$$

Здесь $r_{ \pm}(\alpha), g_{ \pm}(\alpha)$ задаются формулами (7), (8).

ДокАЗАТЕЛьСтво. Доказательство первого утверждения теоремы 1 следует из асимптотик (23). Остальные утверждения - из того факта, что $\tilde{\gamma}_{2}$ является устойчивой инвариантной кривой для минусовой НТ, а кривая $\tilde{\gamma}_{1}-$ устойчивой инвариантной кривой для плюсовой НТ при $\alpha>3 / 2$ и для тривиальной НТ при $1<\alpha \leqslant 3 / 2$.

Покажем, что похожие результаты можно получить для плотности распределения суммарного спина в объеме $\Lambda_{N}$, вычисленной по состоянию, отвечающему термодинамическому пределу и заданному на бесконечномерной алгебре Грассмана, порожденной спинами всех вершин иерархической решетки.

Пусть, например, $\left(r^{\left(N_{0}\right)}, g^{\left(N_{0}\right)}\right) \in \Omega_{1} \cup \Omega_{2}$ для некоторого $N_{0} \geqslant 0$. Из результатов работы [2] следует, что при $\alpha>1$ сушествует термодинамический предел в нашей модели, что означает существование предела всех корреляционных функций $\rho_{N}\left(F\left(\psi^{*}\right)\right)$ при 
$N \rightarrow \infty$. Доказательство сводится к доказательству существования пределов одноточечных корреляционных функций

$$
\begin{aligned}
& u_{N}^{1}(r, g)=\rho_{N}(r, g)\left(\psi_{1}(i) \bar{\psi}_{1}(i)+\psi_{2}(i) \bar{\psi}_{2}(i)\right), \\
& u_{N}^{2}(r, g)=\rho_{N}(r, g)\left(-\psi_{1}(i) \bar{\psi}_{1}(i) \psi_{2}(i) \bar{\psi}_{2}(i)\right), \quad i \in \Lambda_{N} .
\end{aligned}
$$

Пусть

$$
u_{N}(r, g)=\left(\begin{array}{c}
u_{N}^{1}(r, g) \\
u_{N}^{2}(r, g)
\end{array}\right) .
$$

В [2] доказано сушествование предела

$$
u(r, g)=\lim _{N \rightarrow \infty} u_{N}(r, g) .
$$

Кроме того, в случае, если $(r, g)$ при некоторой итерации попадает в $\Omega_{1}$, из результатов работы [2] следует представление

$$
u(r, g)=\sum_{i=0}^{\infty} \prod_{k=0}^{i-1} A\left(r^{(k)}, g^{(k)}\right) s\left(r^{(i)}, g^{(i)}\right)
$$

где

$$
\begin{aligned}
& A(r, g)=\frac{1}{n}\left(\begin{array}{ll}
\frac{\partial r^{\prime}}{\partial r} & \frac{\partial g^{\prime}}{\partial r} \\
\frac{\partial r^{\prime}}{\partial g} & \frac{\partial g^{\prime}}{\partial g}
\end{array}\right), \quad s(r, g)=\frac{1}{n}\left(\begin{array}{l}
\frac{\partial \ln c(r, g)}{\partial r} \\
\frac{\partial \ln c(r, g)}{\partial g}
\end{array}\right), \\
& c(r, g)=\left((r+1)^{2}-g\right)^{n-2}\left((r+1)^{2}-\frac{g}{n}\right) .
\end{aligned}
$$

Пусть $\rho=\lim _{M \rightarrow \infty} \rho_{M}$. Обозначим через $q_{N}^{(a)}\left(x^{*}\right)$ плотность распределения суммарного спина $\psi_{N, a}^{*}$, вычисленную по состоянию $\rho$ :

$$
q_{N}^{(a)}=\rho\left(\delta\left(\psi_{N, a}^{*}-x^{*}\right)\right) .
$$

Легко получить представление

$$
\begin{aligned}
q_{N}^{(1 / 2)}\left(x^{*}\right)= & -n^{2(\alpha-1) N} u_{2}\left(r^{(N)}, g^{(N)}\right)- \\
& -\frac{1}{2} n^{(\alpha-1) N} u_{1}\left(r^{(N)}, g^{(N)}\right)\left(\bar{x}_{1} x_{1}+\bar{x}_{2} x_{2}\right)+\bar{x}_{1} x_{1} \bar{x}_{2} x_{2} .
\end{aligned}
$$

Таким образом, вопрос о предельном поведении плотности $q_{N}^{(1 / 2)}\left(x^{*}\right)$ сводится к такому же вопросу для вектора $D_{N} u\left(r^{(N)}, g^{(N)}\right)$, где

$$
D_{N}=\left(\begin{array}{cc}
\lambda_{1}^{N} & 0 \\
0 & \lambda_{1}^{2 N}
\end{array}\right)
$$


Из (24) получаем представление

$$
D_{N} u\left(r^{(N)}, g^{(N)}\right)=\sum_{i=0}^{\infty} \prod_{k=0}^{i-1} T_{N}\left(r^{(N+k)}, g^{(N+k)}\right) D_{N} s\left(r^{(N+i)}, g^{(N+i)}\right)
$$

где

$$
T_{N}\left(r^{(N+k)}, g^{(N+k)}\right)=D_{N} A\left(r^{(N+k)}, g^{(N+k)}\right) D_{N}^{-1} .
$$

Для матрицы $A$ можно получить удобное представление

$$
A(r, g)=\left(\begin{array}{cc}
\frac{2 n^{\alpha-2}}{n-1}\left(\sigma^{2}-\sigma \frac{n+3}{2}+n\right) & 4 \frac{n^{2 \alpha-3}}{n-1}(r+1) \sigma(1-\sigma)^{2} \\
-\frac{n^{\alpha-1}}{n-1} \cdot \frac{1}{r+1} \cdot\left(1-\frac{\sigma}{n}\right)^{2} & -\frac{2 n^{2 \alpha-4}}{n-1} \sigma\left(\sigma^{2}-\sigma^{3} \frac{n+1}{2}+n\right)
\end{array}\right),
$$

напомним, что

$$
\sigma=\sigma(r, g)=\frac{(r+1)^{2}-g}{(r+1)^{2}-\frac{g}{n}}
$$

Как уже отмечалось, в работе [2] были найдены асимптотики (23), а также то, что $1-\sigma\left(r^{(N)}, g^{(N)}\right)=o\left(n^{-N / 2}\right)$. Отсюда мы получаем

$$
\begin{aligned}
& \lim _{N \rightarrow \infty} T_{N}\left(r^{(N+k)}, g^{(N+k)}\right)=\left(\begin{array}{cc}
0 \\
-\left(1-\frac{1}{n}\right) \frac{n^{\alpha-2}}{c_{1}(r, g)} n^{-k(\alpha-1)} & n^{2(\alpha-2)}
\end{array}\right), \\
& \lim _{N \rightarrow \infty} D_{N} s\left(r^{(N+i)}, g^{(N+i)}\right)=\left(\begin{array}{c}
\frac{2}{c_{1}(r, g)}\left(1-\frac{1}{n}\right) n^{-i(\alpha-1)} \\
-\left(\left(1-\frac{1}{n}\right) \frac{1}{c_{1}(r, g)} n^{-i(\alpha-1)}\right)^{2}
\end{array}\right) .
\end{aligned}
$$

Используя (27) и (28), находим

$$
\lim _{N \rightarrow \infty} D_{N} u\left(r^{(N)}, g^{(N)}\right)=\left(\begin{array}{c}
\frac{2}{c_{1}(r, g)} \\
-\frac{1}{c_{1}^{2}(r, g)}
\end{array}\right) .
$$

Таким образом, имеем

$$
\begin{aligned}
\lim _{N \rightarrow \infty} q_{N}^{(1 / 2)}\left(x^{*}\right) & =\frac{1}{c_{1}^{2}(r, g)}-\frac{1}{c_{1}(r, g)}\left(\bar{x}_{1} x_{1}+\bar{x}_{2} x_{2}+\bar{x}_{1} x_{1} \bar{x}_{2} x_{2}\right)= \\
& =\frac{1}{c_{1}^{2}(r, g)} \exp \left\{-c_{1}(r, g)\left(\bar{x}_{1} x_{1}+\bar{x}_{2} x_{2}\right)\right\},
\end{aligned}
$$

т.е. предел плотностей $q_{N}^{(1 / 2)}\left(x^{*}\right)$ является гауссовской плотностью с “дисперсией” $2 c_{1}(r, g)$.

Предположим теперь, что точка $(r, g)$ принадлежит инвариантной кривой $\tilde{\gamma}_{1}$. В работе [1] были найдены ограничения на $\alpha$ и $n$, при которых сушествует термодинамический предел нашей модели в НТ преобразования РГ. Было показано, что если спектр матрицы $A\left(r_{+}, g_{+}\right)$(соответственно $\left.A\left(r_{-}, g_{-}\right)\right)$лежит внутри единичного круга, то термодинамический предел в плюсовой (минусовой) НТ существует. 
Нетрудно видеть, что при тех же самых условиях на $\alpha$ и $n$ термодинамический предел существует и для точек инвариантных кривых $\tilde{\gamma}_{1}$ и $\tilde{\gamma}_{2}$. В [2] было получено следующее представление для $u_{N}(r, g)$ :

$$
u_{N}(r, g)=\sum_{i=0}^{N-1} \prod_{k=0}^{i-1} A\left(r^{(k)}, g^{(k)}\right) s\left(r^{(i)}, g^{(i)}\right)+\prod_{k=0}^{N-1} A\left(r^{(k)}, g^{(k)}\right) u_{0}\left(r^{(N)}, g^{(N)}\right) \text {, }
$$

где $A(r, g), s(r, g)$ заданы соотношениями $(25),(26)$,

$$
u_{0}(r, g)=\left(\begin{array}{c}
\frac{\partial \ln Z_{0}(r, g)}{\partial r} \\
\frac{\partial \ln Z_{0}(r, g)}{\partial g}
\end{array}\right), \quad Z_{0}(r, g)=\left(\left(r+\frac{1-n^{\alpha-2}}{1+n^{-1}}\right)^{2}-g\right) .
$$

Остановимся для определенности на плюсовой НТ. Если собственные числа матрицы $A\left(r_{+}, g_{+}\right)$лежат внутри единичного круга, то то же самое верно и для матрицы $A(r, g)$, если $(r, g)$ находится достаточно близко к $\left(r_{+}, g_{+}\right)$. Если точка $(r, g)$ находится на кривой $\tilde{\gamma}_{1}$, то начиная с некоторого $k$ спектр матрицы $A\left(r^{(k)}, g^{(k)}\right)$ также будет лежать внутри круга некоторого радиуса $\delta<1$.

Поскольку векторы $s\left(r^{(i)}, g^{(i)}\right), i=1,2, \ldots$, и $u_{0}\left(r^{(N)}, g^{(N)}\right), N=1,2, \ldots$, являются ограниченными по норме некоторой константой, то легко увидеть, что сушествует $\lim _{N \rightarrow \infty} u_{N}(r, g)=u(r, g)$ и для $u(r, g)$ также справедливо представление (24).

Теперь, если мы рассмотрим плотность суммарного спина с нормировкой $\alpha / 2$, то увидим, что

$$
q_{N}^{(\alpha / 2)}\left(x^{*}\right)=-u_{2}\left(r^{(N)}, g^{(N)}\right)-\frac{1}{2} u_{1}\left(r^{(N)}, g^{(N)}\right)\left(\bar{x}_{1} x_{1}+\bar{x}_{2} x_{2}\right)+\bar{x}_{1} x_{1} \bar{x}_{2} x_{2} .
$$

Из разложения

$$
u\left(r^{(N)}, g^{(N)}\right)=\sum_{i=0}^{\infty} \prod_{k=0}^{i-1} A\left(r^{(N+k)}, g^{(N+k)}\right) s\left(r^{(N+i)}, g^{(N+i)}\right)
$$

и того, что

$$
A\left(r^{(N+k)}, g^{(N+k)}\right) \underset{N \rightarrow \infty}{\longrightarrow} A\left(r_{+}, g_{+}\right), \quad s\left(r^{(N+i)}, g^{(N+i)}\right) \underset{N \rightarrow \infty}{\longrightarrow} s\left(r_{+}, g_{+}\right),
$$

мы получаем

$$
u\left(r^{(N)}, g^{(N)}\right) \underset{N \rightarrow \infty}{\longrightarrow} u\left(r_{+}, g_{+}\right)
$$

Тем самым мы доказали, что $\lim _{N \rightarrow \infty} q_{N}^{(\alpha / 2)}\left(x^{*}\right)$ сушествует и является негауссовской плотностью, определяемой константами связи $r_{+}, g_{+}$:

$$
p_{+}\left(x^{*} ; \alpha\right)=-u_{2}\left(r_{+}, g_{+}\right)-\frac{1}{2} u_{1}\left(r_{+}, g_{+}\right)\left(\bar{x}_{1} x_{1}+\bar{x}_{2} x_{2}\right)+\bar{x}_{1} x_{1} \bar{x}_{2} x_{2},
$$

где

$$
u\left(r_{+}, g_{+}\right)=\sum_{i=0}^{\infty}\left(A\left(r_{+}, g_{+}\right)\right)^{i} S\left(r_{+}, g_{+}\right)=\left(I-A\left(r_{+}, g_{+}\right)\right)^{-1} S\left(r_{+}, g_{+}\right)
$$


$I$ - тождественная матрица. Аналогичное рассуждение проходит и для того случая, когда $(r, g)$ принадлежит устойчивой инвариантной кривой $\tilde{\gamma}_{2}$. При этом предельная плотность $p_{-}\left(x^{*}, \alpha\right)$ определяется вектором $u\left(r_{-}, g_{-}\right)$, который находится из соотношения

$$
u\left(r_{-}, g_{-}\right)=\left(I-A\left(r_{-}, g_{-}\right)\right)^{-1} S\left(r_{-}, g_{-}\right) .
$$

Векторы $S\left(r_{ \pm}, g_{ \pm}\right)$и матрицы $A\left(r_{ \pm}, g_{ \pm}\right)$вычисляются явно:

$$
\begin{aligned}
& S\left(r_{ \pm}, g_{ \pm}\right)=\left(\begin{array}{c}
\frac{2}{\sqrt{n} \pm 1} \frac{(n-2 \pm \theta)\left(1 \mp \frac{\theta}{n}\right)}{\sqrt{n}-\theta} \\
\mp \frac{\sqrt{n} \mp 1}{\sqrt{n} \pm 1} \frac{\theta\left(n-2 \pm \frac{\theta}{n}\right)\left(1 \mp \frac{\theta}{n}\right)}{(\theta-\sqrt{n})^{2}}
\end{array}\right), \\
& A\left(r_{ \pm}, g_{ \pm}\right)=\left(\begin{array}{cc}
\frac{2}{(n-1) \sqrt{n} \theta}\left(\theta^{2} \mp \theta \frac{n+3}{2}+n\right) & \pm \frac{4(\sqrt{n}-\theta)(1 \mp \theta)^{2}}{\theta^{2}(n-1)( \pm \sqrt{n}-1)} \\
\mp \frac{\sqrt{n}(\sqrt{n} \mp 1)}{(n-1)(\sqrt{n}-\theta)}\left(1 \mp \frac{\theta}{n}\right)^{2} & \mp \frac{2}{\theta n(n-1)}\left(\theta^{2} \mp \theta \frac{3 n+1}{2}+n\right)
\end{array}\right) .
\end{aligned}
$$

Выполнена следующая теорема.

Tеорема 2. Пусть $2>\alpha>1$. Ecлu $\left(r^{\left(N_{0}\right)}, g^{\left(N_{0}\right)}\right) \in \Omega_{1} \cup \Omega_{2}$ для некоторого $N_{0} \geqslant 0, m o$

$$
\lim _{N \rightarrow \infty} q_{N}^{(1 / 2)}\left(x^{*} ; r, g\right)=p\left(x^{*} ; c_{1}(r, g), 0\right) \text {. }
$$

$\operatorname{Ec} \bumpeq u(r, g) \in \tilde{\gamma}_{2}, m o$

$$
\lim _{N \rightarrow \infty} q_{N}^{(\alpha / 2)}\left(x^{*} ; r, g\right)=p_{-}\left(x^{*} ; \alpha\right)
$$

Ecлu $(r, g) \in \tilde{\gamma}_{1}$, mo при $\alpha>3 / 2$

$$
\lim _{N \rightarrow \infty} q_{N}^{(\alpha / 2)}\left(x^{*} ; r, g\right)=p_{+}\left(x^{*} ; \alpha\right)
$$

а при $1<\alpha \leqslant 3 / 2$

$$
\lim _{N \rightarrow \infty} q_{N}^{(\alpha / 2)}\left(x^{*} ; r, g\right)=p\left(x^{*} ; c_{0}(\alpha), 0\right),
$$

здесь плотности $p_{ \pm}\left(x^{*} ; \alpha\right)$ определяются формулами

$$
p_{ \pm}\left(x^{*} ; \alpha\right)=-u_{2}\left(r_{ \pm}, g_{ \pm}\right)-\frac{1}{2} u_{1}\left(r_{ \pm}, g_{ \pm}\right)\left(\bar{x}_{1} x_{1}+\bar{x}_{2} x_{2}\right)+\bar{x}_{1} x_{1} \bar{x}_{2} x_{2}
$$

$2 \partial e$

$$
\begin{aligned}
u_{1}\left(r_{ \pm}, g_{ \pm}\right) & =\frac{1}{\Delta} \frac{2(n-1)\left(1 \mp \frac{\theta}{n}\right)^{2}}{(\sqrt{n} \pm 1)(\sqrt{n}-\theta)} \\
u_{2}\left(r_{ \pm}, g_{ \pm}\right) & =\mp \frac{1}{\Delta} \frac{(\sqrt{n} \mp 1)^{2}\left(1 \mp \frac{\theta}{n}\right) \theta}{\sqrt{n}(\sqrt{n} \pm 1)(\sqrt{n}-\theta)^{2}}\left(n \pm 2 \sqrt{n}-\theta \frac{2 \sqrt{n} \pm 1}{n}\right), \quad \theta=n^{3 / 2-\alpha} \\
\Delta & =1 \pm n^{-3 / 2}-\frac{2 n^{-3 / 2}}{\left.1 \pm n^{-1 / 2}\right) \theta}\left(\theta^{2} \mp \theta \frac{n}{2}\left(1 \mp n^{-1 / 2}\right)^{2}+n\right) .
\end{aligned}
$$


Таким образом, в случае, если $(r, g)$ принадлежит инвариантным кривым $\tilde{\gamma}_{1}, \tilde{\gamma}_{2}$, предельное поведение суммарного нормированного спина $\psi_{N, \alpha / 2}^{*}$ будет, как и в теореме 1 , негауссовским, хотя и несколько другим.

Эту теорему можно проинтерпретировать как следуюшую картину критического поведения. Зафиксируем значение $r_{0}$. Пусть для определенности $r_{0}>0, \alpha>3 / 2$. Рассмотрим полупрямую $\left(r_{0}, g\right), g>0$. Обозначим через $\left(r_{0}, g_{\text {кр }}\right)$ точку пересечения этой полупрямой с кривой $\gamma_{1}$. Тогда при $0<g<g_{\text {кр }}$ суммарньй спин в объеме $\Lambda_{N}$ с показателем нормировки в пределе $N \rightarrow \infty$ имеет “гауссовское" распределение с “дисперсией":

$$
\chi\left(r_{0}, g\right)=\int\left(x_{1} \bar{x}_{1}+\bar{x}_{2} x_{2}\right) p\left(x^{*} ; c_{1}\left(r_{0}, g\right), 0\right) d x^{*}=\frac{2}{c_{1}(r, g)}
$$

Заметим, что $\chi(r, g)$ можно также записать как

$$
\chi(r, g)=\lim _{N \rightarrow \infty} \rho(r, g) \frac{1}{n^{N}}\left[\sum_{i \in \Lambda_{N}} \psi_{1}(i) \sum_{j \in \Lambda_{N}} \bar{\psi}_{1}(j)+\sum_{i \in \Lambda_{N}} \psi_{2}(i) \sum_{j \in \Lambda_{N}} \bar{\psi}_{2}(j)\right]
$$

и поэтому эту величину можно назвать антикоммутативным аналогом восприимчивости.

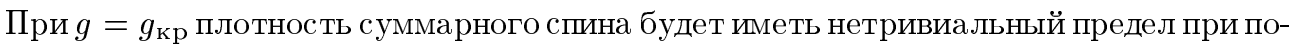
казателе нормировки $a=\alpha / 2$, и этот предел определяется плюсовой “негауссовской" НТ преобразования РГ $p_{+}\left(x^{*} ; \alpha\right)$. При достаточно больших $g$, например при $g>$ $\left(r_{0}+1\right)^{2}$ (как следует из результатов раздела 2$)$, суммарный спин с показателем нормировки $a=1 / 2$ тоже будет иметь в пределе "гауссовское" распределение с "дисперсией" $\chi\left(r_{0}, g\right)=2 c_{1}\left(r_{0}, g\right)^{-1}$, но в отличие от случая $g<g_{c}$ эта “дисперсия" имеет отрицательный знак.

На отрезке от $g_{c}$ до $\left(r_{0}+1\right)^{2}$, как следует из результатов компьютерных экспериментов, мы бесконечно много раз попадаем как в область притяжения инвариантного множества $\Omega_{1}$, так и в область притяжения множества $\Omega_{2}$, а также бесконечно много раз пересекаем устойчивые инвариантные кривые для нетривиальных НТ.

В этом картина критического поведения отличается от картины в “бозонных" моделях, в которых существует единственное значение критической температуры (аналогом обратной температуры в нашей модели можно считать $g$ ).

Компьютерные эксперименты показывают, что, когда $g$ подходит (снизу) к $\tilde{\gamma}_{1}$, дисперсия $\chi\left(r_{0}, g\right)$ стремится к $+\infty$, когда же $g$ подходит (сверху) к $\tilde{\gamma}_{2}$, дисперсия $\chi\left(r_{0}, g\right)$ стремится к $-\infty$; в случае если $g$ пересекает $\tilde{\gamma}_{\infty}$, то $\chi\left(r_{0}, g\right)=0$. График функции $\chi\left(r_{0}, g\right)$ имеет сложный вид с бесконечным числом полюсов.

Рассмотрим вопрос о поведении $\chi\left(r_{0}, g\right)$, когда $g \uparrow g_{\text {кр }}$, где $g_{\text {кр }}-$ ордината пересечения луча $\left(r_{0}, g\right)$ с $\gamma_{1}$. Запишем $g$ в виде $g=g_{\text {кр }}-b(g) \mu_{+}^{k(g)}$, где $\mu_{+}-$старшее собственное число дифференциала РГ в плюсовой НТ, $k(g)$ - натуральное число, $1 \leqslant b(g)<\mu_{+}$. Когда $g \rightarrow g_{\text {кр }}$, то $k(g) \rightarrow \infty$. Напомним, что для дифференциала РГ в плюсовой (минусовой) НТ сушествует явная формула $D\left(r_{ \pm}, g_{ \pm}\right)=n A\left(r_{ \pm}, g_{ \pm}\right)^{\operatorname{tr}}$, где $A\left(r_{ \pm}, g_{ \pm}\right)$задается формулой (29). Если $\alpha>1$, то $\mu_{+}>1$. 
Так как $\chi(r, g)=2 c_{1}(r, g)^{-1}$, то из того, что $c_{1}(r, g)=\lim _{N \rightarrow \infty} r^{(N)} \lambda_{1}^{-N}$, следуют рекуррентные соотношения

$$
\begin{gathered}
c_{1}\left(r^{(k)}, g^{(k)}\right)=\lambda_{1}^{k} c_{1}(r, g), \\
\chi(r, g)=\lambda_{1}^{k} \chi\left(r^{(k)}, g^{(k)}\right) .
\end{gathered}
$$

Рассмотрим предел

$$
\lim _{g \rightarrow g_{\mathrm{kp}}} \frac{\ln \chi\left(r_{0}, g\right)}{\ln \left|g-g_{\mathrm{Kp}}\right|}=\lim _{k(g) \rightarrow \infty} \frac{k(g) \ln \lambda_{1}+\ln \chi\left(r_{0}^{(k(g))}, g^{(k(g))}\right)}{-k(g) \ln \mu_{+}+\ln b(g)} .
$$

Используя методы работы [5], можно показать, что $c_{1}\left(r_{0}^{(k(g))}, g^{(k(g))}\right)<$ const. Отсюда получаем

$$
\lim _{g \rightarrow g_{\mathrm{\kappa p}}} \frac{\ln \chi\left(r_{0}, g\right)}{\ln \left|g-g_{\mathrm{\kappa p}}\right|}=-\frac{\ln \lambda_{1}}{\ln \mu_{+}},
$$

что определяет критический индекс в нашей модели (в бозонных моделях он обозначается как $\gamma)$. Если $r_{0}<-1$, луч $\left(r_{0}, g\right)$ сначала пересекается с кривой $\gamma_{2}$ в некоторой точке $\left(r_{0}, g_{\text {кр }}\right)$. Рассуждая аналогично предыдушему случаю, можно показать, что

$$
\lim _{g \rightarrow g_{\mathrm{Kp}}} \frac{\ln \left|\chi\left(r_{0}, g\right)\right|}{\ln \left|g-g_{\mathrm{Kp}}\right|}=-\frac{\ln \lambda_{1}}{\ln \mu_{-}}
$$

где $\mu_{-}-$старшее собственное число дифференциала РГ в минусовой НТ.

Работа выполнена при финансовой поддержке Российского фонда фундаментальных исследований (грант № 97-01-00375).

\section{Список литературы}

[1] E. Yu. Lerner, M. D. Missarov. J. Stat. Phys. 1994. V. 76. № 3/4. P. 805.

[2] Э. Ю. Лернер, М. Д. Миссаров. ТМФ. 1996. Т. 107. № 2. С. 201.

[3] М. Д Миссаров. ТМФ. 1998. Т. 114. № 3. С. 323.

[4] Ф. А. Березин. Метод вторичного квантования. М.: Наука, 1965.

[5] K. Gawedski, A. Kupiainen. Nucl. Phys. B. 1985. V. 262. P. 33.

Поступила в редакцию 13.IV.1998 г. 